\title{
A Survey of Energy Efficiency in Wireless Human Body Sensors Lifetime for Healthcare Applications
}

ISSN: 2689-2707

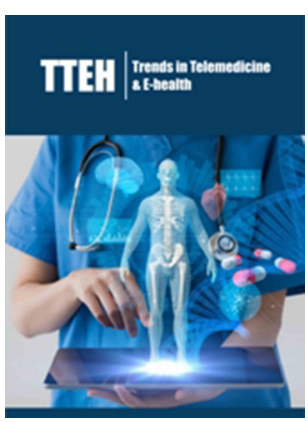

*Corresponding author: Sara Kassan, Department of Computer Science, 34 rue Grillenbreit, 68008 Colmar Cedex, France

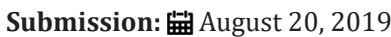

Published: 畺 September 04, 2019

Volume 1 - Issue 5

How to cite this article: Sara K, Jaafar G, Pascal L. A Survey of Energy Efficiency in Wireless Human Body Sensors Lifetime for Healthcare Applications. Trends Telemed E-Health 1(5). TTEH. 000525. 2019. DOI: 10.31031/TTEH.2019.01.000525

Copyright@ Sara Kassan, This article is distributed under the terms of the Creative Commons Attribution 4.0 International License, which permits unrestricted use and redistribution provided that the original author and source are credited.

\author{
Sara Kassan ${ }^{1 *}$, Jaafar Gaber ${ }^{2}$ and Pascal Lorenz ${ }^{1}$ \\ ${ }^{1}$ Department of Computer Science, France \\ ${ }^{2}$ Department of Genie Informatique, France
}

\begin{abstract}
Wireless Human Body Sensor Networks (WHBSNs) are extensively used in vital sign monitoring applications and predicting crop health in in order to identify emergency situations and allow caregivers to respond efficiently. When a sensor is drained of energy, it can no longer achieve its role without a substituted source of energy. However, limited energy in a sensor's battery prevents the long-term process in such applications. In addition, replacing the sensors' batteries and redeploying the sensors can be very expensive in terms of time and budget and need the presence of the patient at the hospital. To overcome the energy limitation, researchers have proposed the use of energy harvesting to reload the rechargeable battery by power. Therefore, efficient power management is required to increase the benefits of having additional environmental energy. This paper presents a review of energy efficient harvesting mechanisms to extend the Wireless Human Body Sensors lifetime.
\end{abstract}

Keywords: Wireless human body sensors; Energy saving mechanisms; Energy efficient harvesting; piezoelectric; Power consumption; Human body

\section{Introduction}

Wireless Human Body Sensor Networks (WHBSNs) used in healthcare systems have widely attracted the attention of the research community [1]. We identify two categories of healthcare-oriented systems: vital status monitoring and remote healthcare surveillance. In vital status monitoring applications, sensors supervise their vital parameters in order to specify emergency situations and allow caregivers to respond efficiently. Applications include disaster monitoring, cancer detection, vital sign monitoring in hospitals, cardiovascular disease and epilepsy seizure detection [2,3]. Remote healthcare surveillance concerns not vital care services for which the continuous healthcare professional is not necessary. For example, Wireless Human Body Sensor nodes are deployed in remote places to sense clinically relevant data information for rehabilitation supervision [4], elderly monitoring [5] or to provide support to a person with cognitive disorder.

Wireless Human Body Sensors transmit the sensing data information to the Base Station (BS) as smartphone or PC that can be connected to a local server or can also be connected to the Internet. Figure 1 shows an example of an architecture of WHBSNs in healthcare applications. Due to the fact that the network lifetime depends on residual energy in the batteries of nodes, energy is one of the extremely important challenge. In this paper, we review the different existing energy efficient mechanisms and wireless energy transfer to prolong the WHBSNs lifetime. The paper is organized as follows: Section 4 defines wireless human body sensors network lifetime and the energy consumption reasons. The aim of Section 5 is to provide a review with different mechanisms to save energy in wireless human body sensors network. In section 6, we present techniques to extract energy harvesting to recompense energy waste in wireless human body sensors network. Finally, we conclude in the paper in section 7.

\section{Wireless Human Body Sensors Network Lifetime}

One of the most critical challenge concerning Wireless Human Body Sensors Network is how to prolong the lifetime of the network. There is not only one definition of lifetime. 
The suitable definition of network lifetime depends on different works presented by researchers in the literature [6]. We present an overview with the major various types used in the literature.

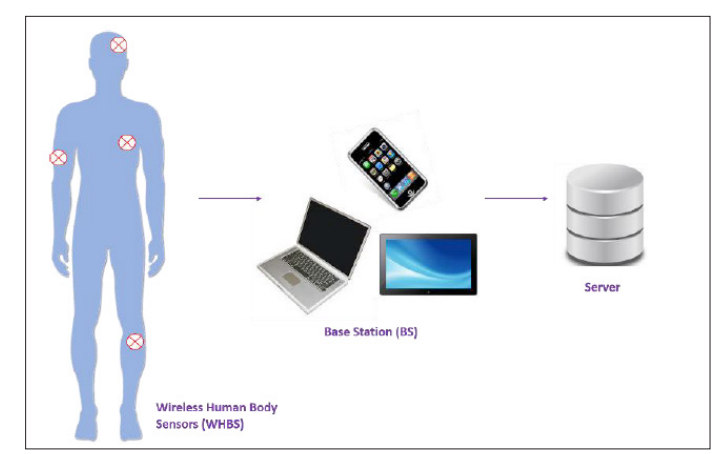

Figure 1: Architecture of wireless human body sensors in healthcare applications.

\section{Network lifetime based on nodes lifetime}

Network lifetime based on the nodes lifetime frequently depends on the time during which all sensor nodes are alive. The sink nodes are excepted from the set of nodes to reflect the statement that sink nodes are more sophisticated and powerful devices. This lifetime can be easily computed since it does not consider the topology changes.

\section{Network lifetime based on interest region coverage}

Coverage is based on the capability of the network to sense a data information in the monitored environment. Therefore, some researchers define the lifetime as the time during which the region of interest is covered by sensors. However, even a high coverage is not sufficient because it does not ensure that collected data information are delivered to the base station.

\section{Network lifetime based on sensor nodes connectivity}

Connectivity reflects the ability of the wireless network to transmit data information to the base station. This definition is similar to what has been proposed in context of ad hoc networks. The different reasons of energy consumption are presented as follows.

Sensing energy consumption: Sensing energy costs depends on the type of sensors. For example, the temperature sensors consumed less important energy than gas sensors. The sensor node can contain diverse sensors, and each one has its individual energy consumption attributes. Generally, the sensing energy consumption for a wireless sensor can be expressed as follows:

$$
E_{s}=\alpha \times N \times V_{d c} \times I_{\text {sens }} \times T_{\text {sens }} \text { (1) }
$$

where $I_{\text {sens }}$ is the intended amount of current, and $T_{\text {sens }}$ is the duration to detect and collect $N$ bits data of information and $\alpha$ is the percentage to compress $N$ bits sensing data information.

Processing energy consumption: The sensor consumes energy to read the data message and to write it in its memory. The processing energy consumption could be calculated by:

$$
E_{p}=\frac{\alpha \times N \times V_{d c}}{8} \times\left(I_{\text {Write }} \times T_{\text {Write }}+I_{\text {Read }} \times T_{\text {Read }}\right)(2)
$$

where $I_{\text {Write }}$ and $I_{\text {Read }}$ are the necessary amount current to write and read one-byte data. $T_{\text {Write }}$ and $T_{\text {Read }}$ are the necessary duration to treat the $L\left(S_{i}\right)$ data information.

Communicating energy consumption: The energy consumed to transmit and receive data messages is computed following the first-order wireless communication model for the radio hardware illustrated in Figure 1; [7].

Transmitter expends energy to turn on the radio electronics and the power amplifier. The energy intended to transmit $L$ bits data message is:

$$
E_{T}=\left\{\begin{array}{l}
\alpha \times N \times E_{\text {elec }}+\alpha \times N \times E_{f s} \times d^{2} \text { whend }<d_{0} \\
\alpha \times N \times E_{\text {elec }}+\alpha \times N \times E_{m p} \times d^{4} \text { whend }>d_{0}
\end{array}\right.
$$

where $E_{\text {elec }}$ denotes the energy dissipated to transmit or receive 1-bit message, the constants $E_{f s}$ and $E_{m p}$ depend on the transmitter amplifier model. $E_{f s}$ is for the free space model, $E_{m p}$ is for multipath model, $d$ is the distance between the transmitter and the receiver and $d_{0}$ represents the threshold distance that can be calculated as follows:

$$
d_{0}=\sqrt{E_{f s} / E_{m p}}
$$

\section{Energy Efficient Mechanisms in Wireless Human Body}

\section{Sensors}

Sensors consume energy while sensing, processing, transmitting or receiving data to achieve the missions required by the healthcare applications. Therefore, researchers have proposed different techniques to increase the WSN lifetime via saving approaches. The main goal of these methods is saving energy. We classify energy efficient mechanisms in wireless human body sensors in Figure 2 as follows.

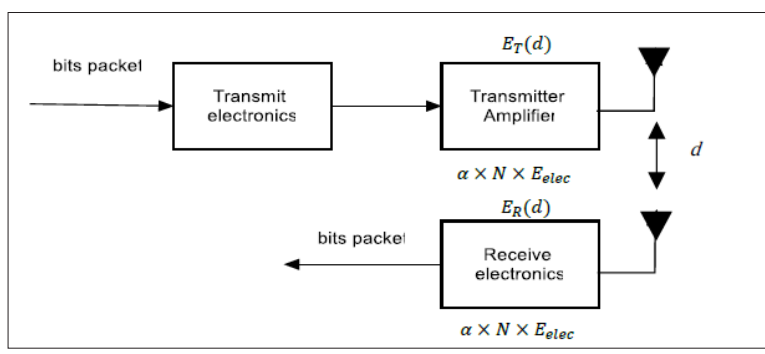

Figure 2: First order radio transceiver model.

\section{Energy routing protocols}

Many routing protocols have been proposed to balance energy consumption so as to extend the WHBSNs lifetime. Energy efficiency routing protocols have been designed with the target of maximizing network lifetime by reducing the energy consumed by the end-to-end transmission and avoiding nodes with low energy in their battery. Some protocols use geographical coordinates of nodes to build a route toward the destination. Others organize a hierarchical structure of nodes to simplify routing and decrease its overhead. Finally, data centric protocols send data information only to interested nodes in order to spare useless transmissions.

Hierarchical based: Hierarchical based routing techniques have advantages in scalability and efficient communication. 
In addition, the concept of hierarchical routing is also used to perform energy-efficient routing in WSNs. In a hierarchical architecture, higher energy sensor nodes can be used to transmit data information, while low energy sensor nodes can be used to perform the sensing in the proximity of the target to conserve energy in the network. The formation of clusters and assigning special responsibilities to cluster heads can significantly contribute to overall network scalability, energy efficiency and lifetime [8]. An adaptive approach for clustering in WSN is proposed in [9]. CHs selection is based on sensor node location and its current residual energy. This approach is powerful in terms of energy dissipation, the number of packets received by the BS and the network lifetime. This approach is more efficient than the known protocol LEACH protocol in terms of energy, number of packets received by the BS and the network lifetime. Data centric or flat routing based: These protocols are query-based and depend on the naming of their data attributes, which help in removing many redundant transmissions. The sink transmits queries to certain areas and waits for data information from the sensor nodes located in the particular areas. Since data information is being requested through queries, attribute-based naming is important to specify the characteristics of data information. Nodes route any data packet by beholding at its content. In the literature, two approaches were proposed. The first one is Sensor Protocol for Information via Negotiation (SPIN) [10] where any node advertises the availability of data and waits for requests from concerned nodes. The second is Directed Diffusion (DD) [11] in which sinks broadcast an important message to sensor nodes and only concerned nodes response with a gradient message. Hence, both interest and gradients establish paths between sink and concerned sensor nodes.

Geographical and energy based: Geographic and Energy Aware Routing (GEAR) [12] protocols use energy-aware and geographically informed neighbor selection heuristics to route a packet toward the destination area. The important idea is to limit the number of interests in directed diffusion by only considering a certain area rather than sending the interests to the whole network. GEAR can save more energy than directed diffusion. Each node in GEAR keeps an estimated cost and a learning cost of reaching the destination through its neighbors. The estimated cost is a combination of residual energy in sensor battery and distance to destination. The learned cost is a refinement of the estimated cost that accounts for routing around holes in the network. A hole occurs when a node does not have any closer neighbor to the target region than itself. If there are no holes, the estimated cost is equal to the learned cost. To conserve more energy, some location-based schemes enable nodes to go to sleeping mode if there is no activity. More energy savings can be obtained by having as many sleeping nodes in the network as possible.

\section{MAC protocols}

The classify Medium Access Control (MAC) layer is one of the most suitable options to achieve better energy efficiency in healthcare application networks [13]. We can classify MAC protocols in three main categories. TDMA-based: for Time Division Multiple Access protocols, time is divided into slots distributed among the sensor nodes. Each slot represented an interval of time to send or receive data information. This technique guarantees a collision free medium access to sensor nodes. It is suitable for periodic traffic [14]. Cascading Information Retrieval by Controlling Access with Dynamic Slot Assignment (CICADA) proposed in [15], is a cross-layered low energy protocol, designed for Time Division Multiple Access (TDMA) scheduling based on multi-hop mobile body area networks. This protocol constructs a spanning tree same. In addition, slots of time are assigned in distributed manner and the identified length of each cycle makes the slot synchronization possible. The parent nodes inform their children nodes when they can communicate. Flow-Aware Medium Access FLAMA [16] is dedicated to data gathering applications. It achieves energy efficiency by reducing idle listening state, avoiding data collisions and transmissions to a sensor node that is not available to receive data packets.

Contention-based: S-MAC tries to force neighbor nodes to adopt the same active/sleep schedule. Therefore, neighbor nodes exchange their programs using SYNC messages sent in the first subperiod. The second subperiod is specified to data exchange. However, listening and sleeping periods cannot be updated after node deployment. For this end, T-MAC [17] enhances S-MAC by allowing nodes to update sleeping time if no message has been received for a fixed time duration. The motivation of D-MAC [18] is to guarantee that all sensor nodes on a multihop path to the sink nodes are awake when the data packet delivery is in progress. D-MAC dynamically selects the preamble sleep/wakeup periods and turns off the nodes to save more energy based on its depth on the forwarding tree. To decrease synchronization overhead, asynchronous sleep/wakeup schemes are based on periodic listening.

Hybrid: This type of protocols switches between TDMA and CSMA protocols to accommodate to variable traffic patterns. One of the most known is Z-MAC protocol [19]. It runs CSMA in low traffic conditions and changes to TDMA in high traffic conditions.

Data reduction: This category aims to reduce the amount of data messages to be delivered to the BS. This category convers the limitation of unnecessary samples and the limitation of sensing tasks because both data transmission and acquisition consume energy.

We can classify data reduction techniques in four types.

Aggregation: In data aggregation structures, nodes along a path towards the sink perform data fusion to reduce the amount of data forwarded towards it. A node can retransmit only the average or the minimum of the received data. Moreover, data aggregation may reduce the latency since it reduces traffic, thus improving delays. However, data aggregation techniques may reduce the accuracy of the collected data. Indeed, depending on the aggregation function, original data may not be recovered by the sink, thus information precision can be lost [20].

Adaptive sampling: The sensing task can be energy-consuming and may generate unneeded samples which affects communication resources and processing costs. Adaptive sampling techniques 
adjust the sampling rate at each sensor ode while ensuring that application requirements are met in terms of coverage or data information precision. In human activity recognition applications, adjust the acquisition frequency to the user activity is proposed that may not be necessary to sample at the same rate when the user is sitting or running [21].

Network coding (NC): These methods are used to decrease the traffic in broadcast scenarios by sending a linear combination of different packets instead of a copy of each packet.

Data compression (DC): These techniques encode data information in such a way that the number of bits required to represent the initial message is reduced. This type of DC is energyefficient because it can reduce transmission times as the packet size is smaller. Data Compression can be divided in two categories: Distributed data compression and local data compression approaches. DC techniques are reviewed in [22].

Topology control: Topology control is another category of energy efficient method that aims to manage the energy consumption of the WSN by suitably adapting the node transmission power and number of neighbors' nodes for maintain good network coverage and connectivity [23].

\section{Energy Harvesting Sources to Recompense Energy Waste in Wireless Human Body Sensors Network}

In the last few years, researchers have proposed different methods to increase the WHBSN lifetime via energy saving approaches as presented in section III. The main goal of these methods is saving energy, during the communication between sensors, without compensation for the energy dissipated via scavenging ambient energy. In this section, we provide recharging batteries based on energy transferred techniques. Figure 3 shows the two approaches to recharge wireless human body sensors' batteries. Recharging batteries methods have extensively attracted the attention of researchers. These two approaches can recharge the battery of WHBSNs without any human intervention.

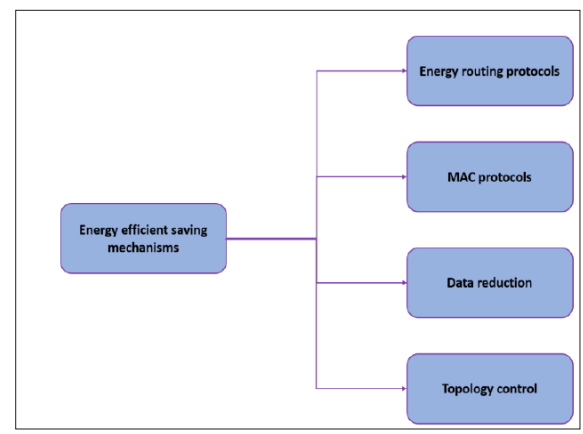

Figure 3: Taxonomy of energy efficient saving mechanisms.

\section{Energy harvesting}

In human body networks, wireless sensor nodes are deployed on or inside of the human body to monitor health evaluation and bodily parameters continuously. Due to their positioning in humans, the nodes need to be operational for long periods of times and preferably for the lifetime of the humans being monitored. Thus, energy can be harvested from humans in different ways, such as through locomotion, blood flow as kinetic power by piezoelectric technique and temperature gradients between the warmer body core and colder areas beneath by thermoelectric generation technique [24,25]. Human body-based energy harvesting can be widely considered as activity-based harvesters and natural physiological parameters-based harvesters. It is essential to note that sensor nodes are considered as energy constrained even during energy harvesting process, so they still require adopting energy conserving approaches.

\section{Wireless energy charging}

The widely development of wireless energy transfer is anticipated to increase the sustainability of WHBSNs. Therefore, wireless energy transfer can be perpetually operational, because these techniques can be utilized to transmit power between wearable devices and can power implantable sensor nodes without any information exchange between sensor nodes and the Base Station (BS). Wireless energy charging in WHBSNs can generally be obtained by electromagnetic (EM) radiation and magnetic resonant coupling methods. However, magnetic resonant coupling emerges to be the most promising technique to address energy requirement of WBSNs, because of its high efficiency at extended distance. One of the actual challenges raised by wireless energy charging technologies is energy cooperation, because it is very vital to transfer energy to neighboring nodes. In future, wireless sensor nodes are intended to have the capacity to harvest energy from the environment and to be efficient in multi-hop energy transferring to other sensor nodes that cannot harvest energy from their environment, to build an autonomous network (Figure 4).

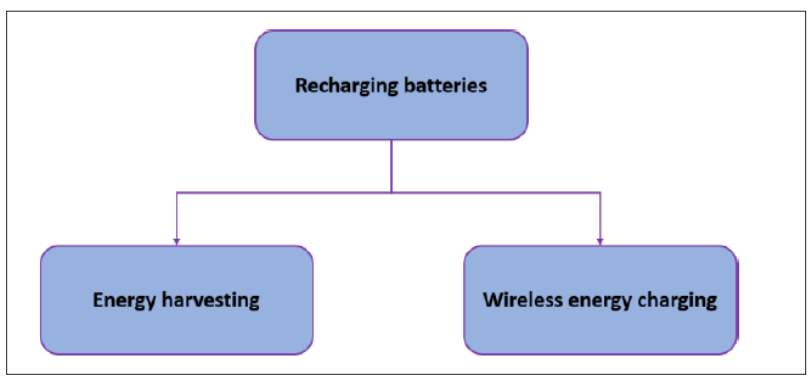

Figure 4: Classification of recharging batteries techniques for WHBSNs.

\section{Conclusion}

This paper summarizes the different existing techniques that tackle the energy efficiency challenge in WHBSNs. The fact that energy saving mechanisms encourage the research on looking for the development of hybrid energy efficient and recharging battery friendly solutions, which guarantee low power consumption and longer battery lifetime in WHBSNs.

\section{References}

1. Ullah HS, Higgins H, Braem B, Latre B, Blondia C, et al. (2012) A comprehensive survey of wireless body area networks. Journal of Medical Systems 36(3): 1065-1094. 
2. Khan RA, Pathan AK (2018) The state-of-the-art wireless body area sensor networks: A survey. International Journal of Distributed Sensor Networks, USA

3. Liu J, Chen Y, Wang Y, Chen X, Cheng J, et al. (2018) Monitoring vital signs and postures during sleep using Wi-Fi signals. In IEEE Internet of Things Journal 5(3): 2071-2084.

4. Hadjidj A, Souil M, Bouabdallah A, Challal Y, Owen H (2013) Wireless sensor networks for rehabilitation applications: Challenges and opportunities. Journal of Network and Computer Applications 36(1): $1-15$.

5. Ali M, Ali AA, Taha A, Dhaou IB, Gia TN (2019) Intelligent autonomous elderly patient home monitoring system. ICC 2019- 2019 IEEE International Conference on Communications (ICC), Shanghai, China, pp. 1-6.

6. Dietrich I, Dressler F (2009) On the lifetime of wireless sensor networks. Journal ACM Transactions on Sensor Networks (TOSN) 5(1).

7. Heinzelman WR, Chandrakasan A, Balakrishnan H (2000) Energyefficient communication protocol for wireless microsensor networks. Hawaii International Conference on System Sciences, Hawaii, USA.

8. Manap Z, Ali BM, Ng CK, Noordin K, Sali A (2013) A review on hierarchica routing protocols for wireless sensor networks. Wireless Personal Communications 72(2): 1077-1104.

9. Kassan S, Lorenz P, Gaber J (2018) Low energy and location-based clustering protocol for wireless sensor network. IEEE International Conference on Communications (ICC), Kansas City, MO, USA, pp. 1-6.

10. Woodrow E, Heinzelman W (2002) SPIN-IT: A data centric routing protocol for image retrieval in wireless networks. ICIP (3): 913-916.

11. Intanagonwiwat C, Govindan R, Estrin D, Heidemann J, Silva F (2003) Directed diffusion for wireless sensor networking. IEEE/ACM Transactions on Networking (TON) 11(1): 2-16.

12. Yu Y, Estrin D, Govindan R (2001) Geographical and energy-aware routing: A recursive data dissemination protocol for wireless sensor networks. UCLA Comp Sci Dept tech, USA.

13. Anand GS, Jong TP (2010) Energy-efficient MAC protocols for wireless body area networks: Survey. International Congress on Ultra-Modern Telecommunications and Control Systems, Moscow, Russia, pp. 739-744.
14. Bilandi N, Verma HK, Dhir R (2018) An energy efficient TDMA based mac protocol for wireless body. $1^{\text {st }}$ International Conference on Secure Cyber Computing and Communication (ICSCCC), Jalandhar, India, pp. 545-549.

15. Latre B, Bart B, Ingrid M, Chris B, Elisabeth R, et al. (2007) A lowdelay protocol for multihop wireless body area networks. $4^{\text {th }}$ Annual International Conference on Mobile and Ubiquitous Systems: Networking \& Services (MobiQuitous), Philadelphia, USA, pp. 1-8.

16. Rajendran V, Garcia LAJJ, Obraczka K (2005) Energy-efficient, application-aware medium access for sensor networks. IEEE International Conference on Mobile Adhoc and Sensor Systems Conference, Washington DC, USA, pp. 8-630.

17. Ram M, Kumar S, Kumar V, Sikandar A, Kharel R (2019) Enabling green wireless sensor networks: Energy efficient t-mac using markov chainbased optimization. Electronics 8(5): 534.

18. Lu G, Krishnamachari B, Raghavendra CS (2004) An adaptive energy efficient and low-latency Mac for data gathering in wireless sensor networks. In Proc $18^{\text {th }}$ International Parallel and Distributed Processing Symposium, New Mexico, USA, pp. 26-30.

19. Rhee I, Warrier A, Aia M, Min J (2005) Z-MAC: A hybrid MAC for wireless sensor networks. In Proc ACM SenSys, S Diego, USA.

20. Padmaja P, Marutheswar GV (2018) Energy efficient data aggregation in wireless sensor networks. Materials Today: Proceedings 5(1): 388-396.

21. Anastasi G, Conti M, Francesco MD, Passarella A (2009) Energy conservation in wireless sensor networks: A survey. Ad Hoc Networks $7(3)$ : 537-568.

22. Kimura N, Latifi S (2005) A survey on data compression in wireless sensor networks. International Conference on Information Technology: Coding and Computing (ITCC'05) 2, Las Vegas, USA, pp. 8-13.

23. Choi SD (2006) A low-power star-topology body area network controller for periodic data monitoring around and inside the human body. In Proc International Symposium on Wearable Computers (ISWC), pp. 139-140.

24. Narita F, Fox M (2017) A review on piezoelectric, magneto strictive, and magnetoelectric materials and device technologies for energy harvesting applications. Adv Eng Mater 20(5).

25. Kassan S, Gaber J, Lorenz P (2019) Autonomous energy management system achieving piezoelectric energy harvesting in wireless sensors. Mobile Networks and Applications, pp. 1-12. 